\title{
The Use of Fingerprint Contact Area for Biometric Identification
}

\author{
M.B. Edwards, G.E. Torrens, and T.A. Bhamra \\ Extremities Performance Research Group, Department of Design and Technology, \\ Loughborough University, Loughborough, LE11 3TU, UK \\ m.b.edwards@lboro.ac.uk
}

\begin{abstract}
This paper details the potential use of finger contact area measurement in combination with existing fingerprint comparison technology for the verification of user identity. Research highlighted includes relationships between finger contact area, pressure applied and other physical characteristics. With the development of small scale fingerprint readers it is starting to be possible to incorporate these into a wide range of technologies. Analysis of finger pressure and contact area can enhance fingerprint based biometric security systems. The fingertip comprises a range of biological materials which give it complex mechanical properties. These properties govern the way in which a fingertip deforms under load. Anthropometric measurements were taken from 11 males and 5 females along with fingerprint area measurements. Strong correlations were found between fingerprint area and many other measurements, including hand length. Notably there were more strong correlations for the female group than for the male. This pilot study indicates the feasibility of fingerprint area analysis for biometric identification. This work is part of a long term program of human physical characterization.
\end{abstract}

\section{Introduction}

This paper details the potential use of finger contact area measurement in combination with existing fingerprint comparison technology for the verification of an individual's identity. Details of current knowledge in the field provide an indication of the feasibility of using enhanced fingerprint technology in this way. The information highlighted includes relationships between finger contact area, pressure applied and other physical characteristics.

\section{Fingerprinting Technology}

Fingerprinting is a known technology with well established protocols for fingerprint comparison. With the development of small scale fingerprint readers it is starting to be possible to incorporate these into security systems and products. The small silicone-based sensors are now compact enough to fit into hand-held devices.

However the use of fingerprint matching technology opens up the possibility of abuse of the system. A number of techniques have been developed that can be used in 
conjunction with fingerprinting to improve its accuracy. These techniques use metrics such as temperature, conductivity and pulse measurement to check the finger placed upon a sensor is from a living person [1]. While these do improve the fallibility of fingerprinting, all of these methods can be circumvented. For example, checks upon the temperature of the finger can be falsified using a thin silicone rubber cast of the desired fingerprint placed upon a finger. This will be kept at the correct temperature by the underlying finger and have the correct pattern of ridges to deposit the required fingerprint.

\section{Fingertip Deformation Prediction}

Consideration of the tissues of the fingertip shows that the analysis of finger pressure and contact area can prevent the use of fake fingerprints for the accessing of protected system. The different tissues within the fingertip give it complex mechanical characteristics which are dependent upon a number of different factors, including size, rate and direction of force application [2]. This allows the fingertip to attenuate small forces applied and transmit larger forces to the underlying bones so making it an effective tool for both exploratory and manipulative tasks. Deformation of these tissues occurs when the fingertip is pressed against a surface and the amount of deformation dictates the size of the fingerprint deposited.

Non-linear viscoelastic theory has been used by a number of researchers to model the deformation of the finger. These models do not accurately predict the changes to the separate materials within the fingertip, instead considering it as one homogeneous material. These models have been found to be accurate in predicting a variety of factors such as plastic distortion of the skin [3], force displacement during tapping [4] and the mechanical responses of the fingertip to force application during dynamic loading $[5,6]$.

All of these fingertip models use information about the physical properties of the finger including its size, elasticity and viscosity to predict the manner in which the fingertip deforms. These physical properties are treated as constants while size and force applied are variables in the models. As such, knowledge of finger size and applied force should allow for the prediction of fingerprint area. The force applied can be measured using transducers placed within a fingerprint scanner. This leaves the deposited fingerprint size as a variable through which one person can be distinguished from another.

\section{$4 \quad$ Fingertip Size}

Anthropometric surveys conducted in the UK have shown fingertip dimensions vary across the population. Index finger depth at the distal joint has been found to vary between $12.5 \mathrm{~mm}$ and $15.1 \mathrm{~mm}$ while its breadth varies between $16.5 \mathrm{~mm}$ to $17.1 \mathrm{~mm}$ [7]. No link has been found between the pattern of ridges on a fingertip and body size. The range of sizes across the population makes finger size a useful measurement for validating a deposited fingerprint. As fingertip size influences the contact area between the fingertip and a object, it will be a component factor in a model predicting 
fingerprint area. If finger size is measured when a fingerprint is first entered into a database, the deposited fingerprint area can be calculated using a suitable model each time the fingerprint is read and used for validation of the entered print.

For the validation of a model for fingertip deformation, Serina et al [8] performs some preliminary tests of finger contact areas for a range of finger forces. In this testing, all forces were subject generated at specified forces between $0.25 \mathrm{~N}$ and $5 \mathrm{~N}$. The forces were held for 2 seconds and the contact area measured by inking the finger before the test and measuring the resultant fingerprint. The author then nondimensionalised the data by dividing the contact areas by the square of the finger width. The nondimensionalised data shows a rapid increase in contact area below $1 \mathrm{~N}$, after which the area steadily in relation to force. This shows that the contact area of the finger for a set force is repeatable and should be modelable. While the authors own predictive model appears to be a poor fit for the data, it purpose is mainly to model finger displacement, with contact are as a extra output. By focusing purely upon contact area, it should be possible to produce a better model. Dividing the data by the square of the finger width removes the main effects of finger size within this figure. This illustrates the basic relationship between force and contact area and gives a indication of the importance of finger size.

\section{Body Size Proportionality}

Another possible further application of this idea is the use of the proportionality of the human body to attempt to predict an approximate body size or weight from a fingerprint. This can then be compared to other measurement of the individual whose fingerprint is being taken, such as height or weight.

Attempts to define the proportion of the human body have been made for centuries, many by artists in order to produce realistic figure drawings. These art-based methods often define the proportion of the body using a limb length as a unit of distance through which the rest of the body can be measured, essentially defining the body as being proportional in size. For example, stature is often defined as being eight times the distance from the chin to the top of the head. More recent anthropometric studies have shown that many individual anatomical measures of the body are correlated and that the human body does indeed have a degree of proportionality. Roebuck [9] gives the correlation values for a range of anthropometric measurements of both U.S. civilian males and females. For both of these groups there are a number of strong correlation coefficients, which indicate proportionality within the human body. For example many bone lengths are strongly correlated as are many limb girths with weight.

\section{Fingerprint Area Investigation}

In order to investigate the relationship between fingerprint area and other body characteristics a survey of $17(n=11$ male, $n=5$ female) students at Loughborough University, UK, was conducted. This study measured both male and female students although analysis of these was conducted separately as size and geometry differences have been found between male and female hands [10].

Fingerprint area was measured by applying a $10 \mathrm{~N}$ load to the back of an inked finger, which pressed the finger against a sheet of photocopy paper. The load was applied by a moving platen held within a guiding frame which ensured the force was 
applied perpendicularly to the back of the finger. The area of the resultant fingerprint was then measured using a planimeter. For comparison with the fingerprint area, each of the participants had nine anthropometric measurements taken. All length measurements were taken with either digital calipers or a Holtain anthropometer depending on the size of measurement to be taken. Height was taken using a portable stadiometer to the nearest millimeter and weight using digital weighing scales, accurate to the nearest half kilogram.

\subsection{Results}

Correlations were produced for all measurements against fingerprint area (see Table 1) and those with a high correlation (Pearsons $r>0.65$ ) were noted. These illustrated a correlation of fingerprint area with a number of measurements, including fingertip length,

Table 1. Correlation coefficients between various anthropometric measurements and fingerprint area

\begin{tabular}{l|r|r} 
& \multicolumn{2}{|c}{ Fingerprint areas } \\
& Male & Female \\
\hline Stature & 0.70 & -0.22 \\
Weight & 0.64 & 0.85 \\
Arm length & 0.68 & 0.64 \\
Hand length & 0.83 & 0.81 \\
Hand width & 0.76 & 0.90 \\
Finger tip length & 0.76 & 0.79 \\
Finger tip width & 0.52 & 0.95 \\
Finger tip Depth & 0.28 & 0.88 \\
Finger tip diameter & 0.26 & 0.81
\end{tabular}

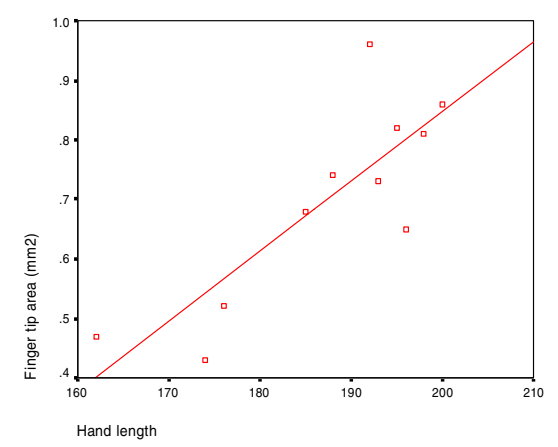

Fig. 1. Scatter plot of fingerprint area against hand length

hand length, arm length and height. Interestingly, there were a larger number of high correlations for the female measurements than then male measurements with the no- 
table exception of height, which is the only negative coefficient. It is thought that this is due to an erroneous height measurement which will have a large effect on the small sample group. Scatter plots of the high correlations were created to confirm that these correlations were not erroneous, and example of which can be seen in Figure 1.

\section{Discussion}

Fingerprinting is the most commonly used biometric security method; however it is not without its problems. The consideration of fingertip structure shows there is a relationship between finger contact area, pressure applied and finger size. This knowledge can be used to enhance current fingerprint security by incorporating it into existing fingerprinting technology. In addition to this, possible links between fingerprint area and body size may allow for a larger increase in the security of fingerprint protected devices.

In order for fingerprint area measurement to become a successful security system, it is important to have an accurate method of measuring the contact area of a finger placed upon a sensor.

A number of laboratory based area measurement techniques have been evaluated by the authors. These all measured the area of an inked fingerprint and included manual techniques involving graph paper, different types of planimeters and a computer program written specifically for the task which was used a scanner to digitise the fingerprint. All of these methods were found to be reliable and repeatable apart from the fully automatic program. This was due to the variability of the amount of ink deposited by the finger. An excess of ink upon the finger makes a much darker fingerprint and this influenced the measurement made by this computer system. The other techniques were not affected by this as they all involved human judgment being used to define the edges of the fingerprint.

The influence of the amount of ink on the finger upon the automatic measurement illustrates some of the problems which may be encountered with a system that is to be used outside of a controlled laboratory. Environmental factors, such as dirt, oil and moisture may have a similar influence to ink for an automatic system, making the fingerprint appear bigger. These are examples of a few environmental factors that require consideration.

The physiological condition of the finger is also a matter that requires consideration. A number of factors can change the mechanical properties of finger tissues and this will affect its deformation. Temperature affects the rigidity of many of the tissues in the body, sweat will make the skin more flexible and stress will affect the levels of sweat produced upon the palm.

From existing literature and the development of the procedure for the tests described in the previous section, a number of different issues were found to be important. These are shown in Figure 2. Many of the issues identified were kept constant, however preliminary testing was done to acertain the effects of variations in the angle of the finger how far along the length of the finger was considered a print of the fingertip. These both were found to have a large effect upon the results. To remove these effects, they were controlled by keeping hand posture the same for each measurement and ensuring only the fingertip above the distal interphalangeal joint was in contact with the paper. These factors all require further investigation before fingerprint area measurement can be used to. 


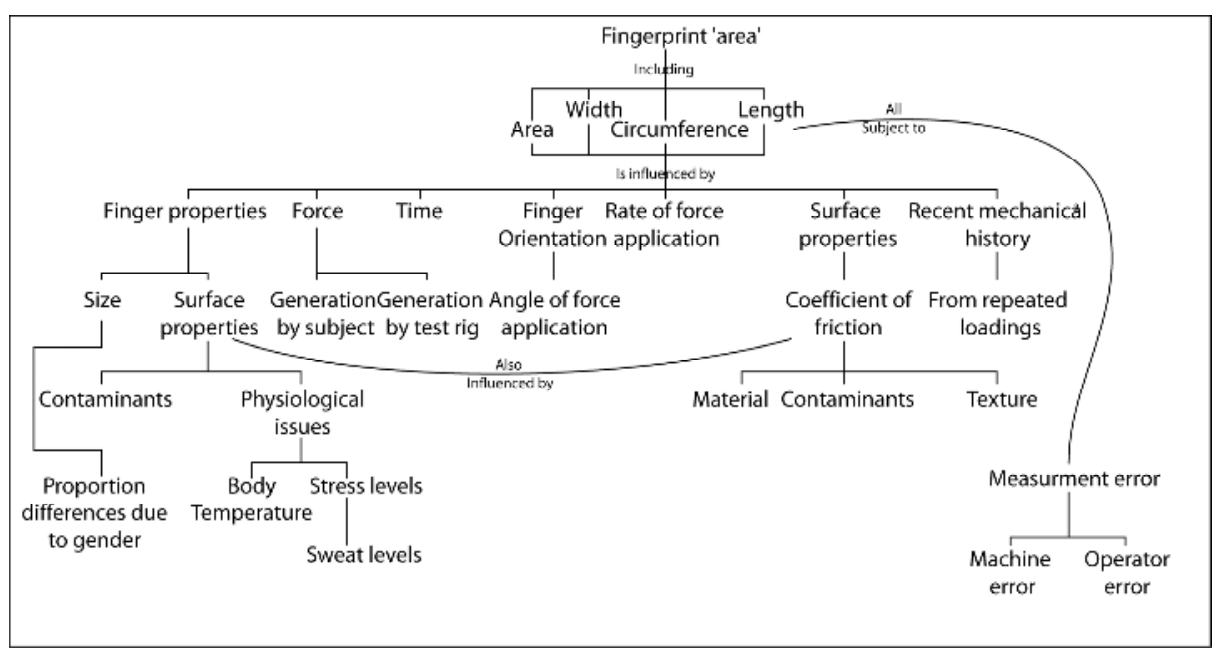

Fig. 2. Issues found to be relevant for fingerprint area deposition

As these factors are addressed, it should be possible to begin to use fingerprint area measurement to enhance biometric security systems through the development of an accurate model predicting fingertip deformation. In order to take this idea from being a concept, to a proven method, for fingerprint-based security augmentation a number of stages of research are planned.

\section{Conclusions}

The use of fingerprint area measurement provides a new method for augmenting fingerprint recognition. This can be potentially applied within numerous security systems due to the size of the sensors required. Before it can be applied, there are a number of issues that need to be addressed, including the effects of a number of factors upon fingerprint area, the production of a model predicting fingerprint deformation and the accuracy of the method used for fingerprint area measurement. Work is currently being performed to address these issues and bring this concept closer to being a usable technique for augmenting fingerprint based security.

A more in-depth investigation into the relationship between fingertip size and deposited fingerprint is currently planned. This will involve the use of a range of sizes of fingertips, a range of force applications and rates of force applications. With these relationships known, a pragmatic model of the fingertip and its deposition area is to be developed. This model will attempt to allow for the determination of fingertip size from a deposited fingerprint at a known load and so not model the deformation of the fingertip. Once this is completed other factors shown in Figure 2 will be investigated to broaden the model. 


\section{References}

1. Biometric Technology Today (2001). Forging Ahead. 9, 9-11

2. Serina, E. R., Mote Jr, C. D. and Rempel, D. (1997). Force response of the fingertip pulp to repeated compression - effects of loading rate, loading angle and anthropometry. Journal of biomechanics, 30, 1035-1040.

3. Cappelli, R., Maio, D. and Maltoni, D. (2001). Modelling Plastic Distortion in Fingerprint Images. In Second International Conference on Advances in Pattern Recognition (ICAPR2001)Rio de Janeiro, pp. 369-376.

4. Jindrich, D., Zhou, Y., Becker, T. and Dennerlein, J. (2003). Non-linear viscoelastic models predict fingertip pulp force-displacement characteristics during voluntary tapping. Journal of Biomechanics, 36, 497-503.

5. Wu, J. Z., Dong, R. G., Smutz, W. P. and Rakheja, S. (2003a). Dynamic interaction between a fingerpad and a flat surface: experiments and analysis. Medical Engineering \& Physics, 25, 397-406.

6. Wu, J. Z., Dong, R. G., Smutz, W. P. and Schopper, A. W. (2003b). Modelling of timedependant force response of fingertip to dynamic loading. Journal of Biomechanics, 36, 383-392.

7. Department of Trade and Industry (1998) Adultdata: The handbook of Adult Anthropometric and strength measurements - Data for Design Safety. Institute for Occupational Ergonomics, Nottingham.

8. Serina, E. R., Mockenstrum, E., Mote Jr, C. D. and Rempel, D. (1998). A structural model of the forced compression of the finger pulp. Journal of Biomechanics, 31, 639-646.

9. Roebuck, J. A. (1995) Anthropometric Methods: Designing to Fit the Human Body. Human Factors and Ergonomics Society, Santa Monica.

10. Rahman, Q. and Wilson, G. D. (2003). Sexual orientation and the 2nd to 4th finger length ratio: evidence for organising effects of sex hormones or developmental instability? Psychoneuroendocrinology, 28, 288-303. 